\title{
Optical turbulence above mountains seen in 3D
}

S. G. Els, K. Vogiatzis, A. Otárola, R. Riddle, M. Schöck, et al.

S. G. Els, K. Vogiatzis, A. Otárola, R. Riddle, M. Schöck, W. Skidmore, T. Travouillon, "Optical turbulence above mountains seen in 3D," Proc. SPIE 7733, Ground-based and Airborne Telescopes III, 77331Q (5 August 2010); doi: $10.1117 / 12.856304$

Event: SPIE Astronomical Telescopes + Instrumentation, 2010, San Diego, California, United States 


\title{
Optical turbulence above mountains seen in 3D
}

\author{
S.G. Els ${ }^{a, c}$ K. Vogiatzis ${ }^{b}$ A. Otárola ${ }^{b}$ R. Riddle ${ }^{b, d}$ M. Schöck ${ }^{b}$ W. Skidmore ${ }^{b}$ T. Travouillon ${ }^{b}$ \\ ${ }^{a}$ Gaia DPAC Project Office, ESAC, PO Box 78, 28691 Villanueva de la Cañada, Madrid, Spain \\ ${ }^{b}$ TMT Observatory Corporation, 2632 E. Washington Blvd., Pasadena, CA 91107, USA \\ ${ }^{c}$ Cerro Tololo Inter-American Observatory, Casilla 603, La Serena, Chile \\ ${ }^{d}$ Caltech Optical Observatories, Caltech, 1200 E. California Blvd, Pasadena, CA 91125, USA
}

\begin{abstract}
Atmospheric optical turbulence is the main driver of wavefront distortions which affect optical telescope performance. Therefore, many techniques have been developed to measure the optical turbulence strength along the line of sight. Based on data collected with the MASS (Multi Aperture Scintillation Sensor), we show that a large sample of such measurements can be used to assess the average three dimensional turbulence distribution above ground. The use of, and a more sophisticated instrumental setup for, such turbulence tomography will be discussed.
\end{abstract}

Keywords: Site testing, optical turbulence profiling, atmospheric turbulence

\section{INTRODUCTION}

With the wide distribution of the Multi Aperture Scintillation Sensor (MASS ${ }^{1}$ ) the monitoring of the vertical distribution of optical turbulence has become a routine operation at most of the current and future astronomical observatory sites. Despite its weakness of only measuring optical turbulence profiles with low vertical resolution, its ability to operate on small aperture telescopes $(\sim 20 \mathrm{~cm})$ and using single stars as targets makes MASS an extremely effective technique. The combination of MASS with the classical Differential Image Motion Sensor $\left(\mathrm{DIMM}^{2}\right)$ in a single instrument package ${ }^{3}$ is being used in all site testing campaigns for the various Extremely Large Telescope projects. These campaigns have resulted in a huge amount of measured vertical turbulence profiles above numerous sites around the world.

The collected data are commonly corrected for the zenith distance. This way the data are interpreted as the typical vertical distribution of the optical turbulence above the site, i.e., towards zenith. However, in reality the data are collected mostly by observing stars under the zenith angle $z=\left(90^{\circ}\right.$-alt $)$; where alt is the altitude (or elevation) of the star above the horizon at the time of observation. That the statistical properties of the turbulence profiles and of the total seeing are indeed representing the conditions towards zenith is based on the assumption that the turbulence strength of the atmospheric layers is on average horizontally uniform. While this assumption is reasonable and has been quantified on an isolated mountaintop, ${ }^{4}$ uniformity - even on average - is not to be expected above more complex surrounding terrains. Indications for inhomogeneities have been found so far in numerical studies and observations covering short periods. ${ }^{5}$ But if, for example, an observing site is below the summit one might expect some directional dependencies in the turbulence strength to be detectable. In this paper we take a closer look at a situation in which a turbulence profiler was located below the summit of Mauna Kea and investigate the impact of the presence of that summit on the optical turbulence strength across the sky as seen from that location.

Further author information: (Send correspondence to S.G.E.)

S.G.E.: E-mail: sels@sciops.esa.int

Ground-based and Airborne Telescopes III, edited by Larry M. Stepp, Roberto Gilmozzi, Helen J. Hall Proc. of SPIE Vol. 7733, 77331Q - (C) 2010 SPIE · CCC code: 0277-786X/10/\$18 · doi: 10.1117/12.856304 


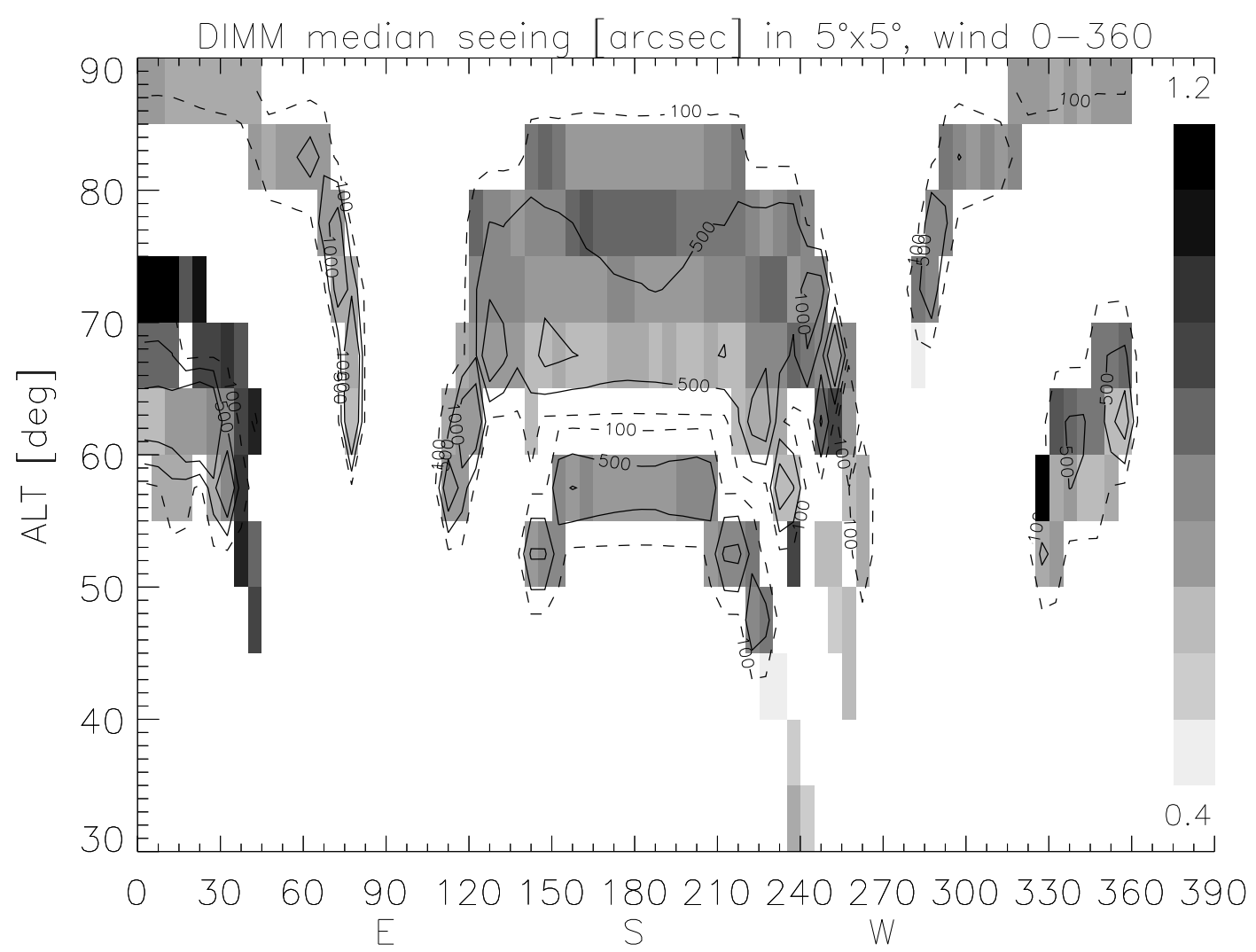

Figure 1. Skymap of median DIMM seeing. The gray scale bar on the right indicates the median DIMM seeing (towards zenith) in arcsec. Dashed contours indicate the regions in which more than 100 observations per pixel were collected. Solid contours indicate areas with more than 500 and 1000 observations per field. The graph contains all data collected at $13 \mathrm{~N}$, i.e., no wind direction selection was done.

\section{MASS-DIMM DATA FROM 13N}

The Thirty Meter Telescope project ${ }^{6}$ (TMT) has decided on the location $13 \mathrm{~N}$ on Mauna Kea (Hawaii) as the preferred location where this future facility shall be erected. The $13 \mathrm{~N}$ site is approximately $150 \mathrm{~m}$ below and $1.6 \mathrm{~km}$ north of the summit ridge of Mauna Kea. Between mid 2005 and mid 2008 the TMT site testing project ${ }^{7}$ operated its T6 site monitoring system at $13 \mathrm{~N}$. This system contained a wide variety of turbulence monitoring devices. The core of that system was a telescope equipped with a MASS-DIMM device. Both channels, the MASS and DIMM, operated in parallel measuring light from the same star and measured the turbulence parameters at a wavelength of $500 \mathrm{~nm}$. For more technical details on this setup we refer to the TMT site testing articles. ${ }^{7}$

\section{THE DIMM SEEING ACROSS THE SKY ABOVE 13N}

The DIMM channel measures the variance of the separation of two images of the same star taken through two sub-pupils of the site monitoring telescope. The variation of the image separation is a direct measure of the total seeing from the telescope to the top of the atmosphere. ${ }^{2}$ Applying the $(\sec z)$ correction normalizes the measurement taken under a non-zero zenith angle to a zenith seeing value. But again, these observations trace air which is seen under a certain altitude and azimuth (alt,az) from the site. The normalization to zenith, however, does not prevent us to compare the turbulence strengths along various lines of sight; it is in fact necessary. In Fig. 1 we show a skymap of the median seeing. To create this map we divided the sky in $5^{\circ} \times 5^{\circ}$ (alt,az) fields and identified all DIMM data obtained while pointing in each of the respective (alt,az) fields. We then 

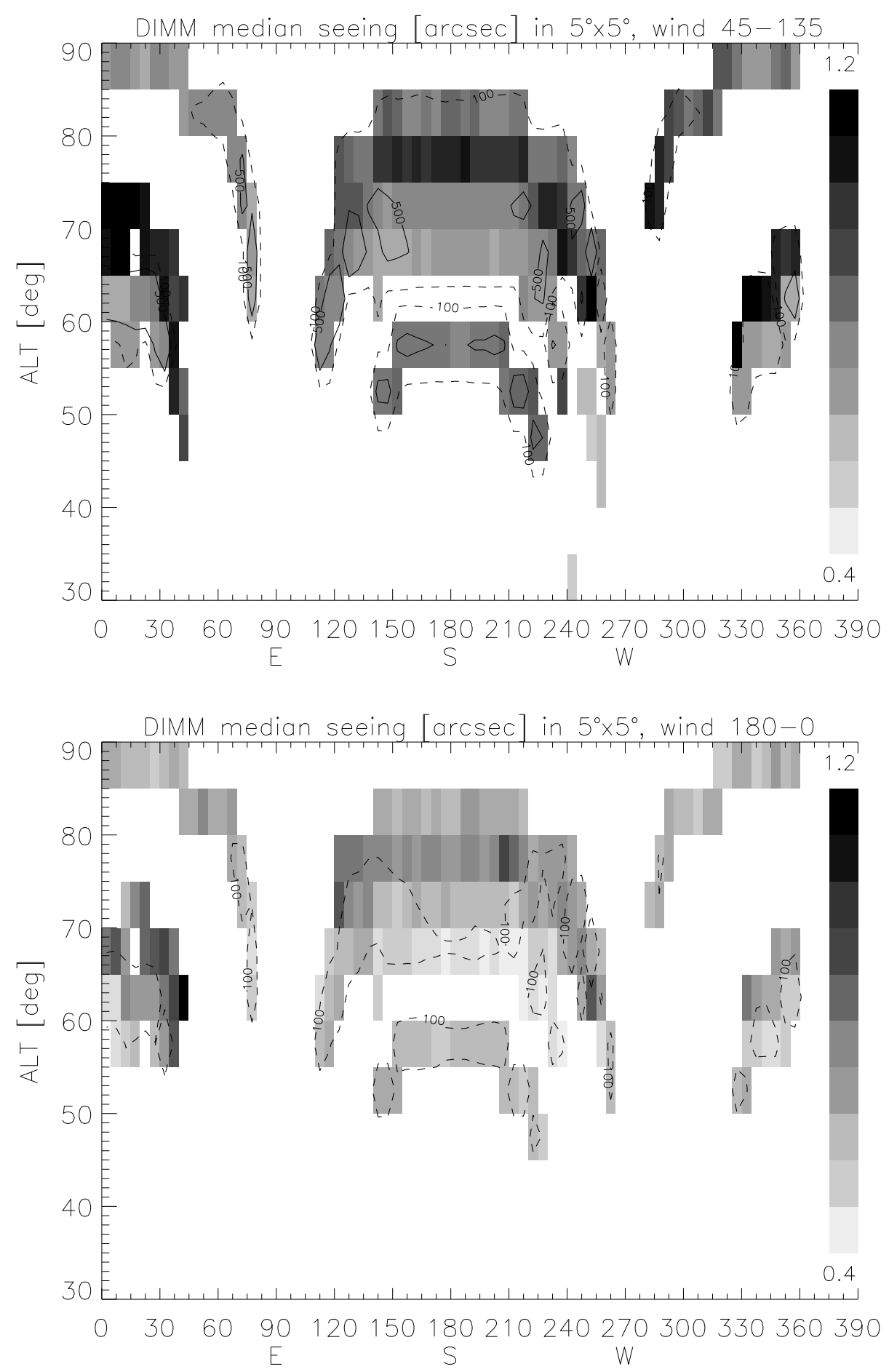

Figure 2. Skymaps of median DIMM seeing, similar to Fig. 1. These figures show only data collected during particular wind conditions. Top: Only DIMM data collected during times when winds were coming from the prevailing wind directions, i.e., $45^{\circ}-135^{\circ}$. Bottom: Data collected when the wind originates between $180^{\circ}$ and $360^{\circ}$. 
computed for each field the median DIMM seeing. The map in Fig. 1 covers the time period between July 2005 and June 2008, containing a total of 134, 208 measurements of each, MASS, DIMM and weather station data taken simultaneously (within $60 \mathrm{sec}$ of each other). The amounts of data contained in the fields are outlined by the contours. During a typical night 250 observations were obtained by following three to five stars. This implies that each field has been visited during several nights and the map in Fig. 1 does not represent any particular weather pattern.

From Fig. 1 it is obvious that the sky has not been covered homogeneously. For practical reasons an on average - homogeneous coverage is very difficult to obtain and was not considered in the definition of the observing scheme and the selection of target stars. We believe that no site monitor is presently operated in a way to cover the sky homogeneously. Thus, certain areas have been covered much more than others. In Fig. 1 the region between az $\approx 320^{\circ}$ to $40^{\circ}$ and alt $\approx 70 / 75^{\circ}$ to $40 / 45^{\circ}$ stands out, as it is the area of strongest median seeing. But this area contains very few observations $(<100)$ and represents only few hours of observations.

The best observed area is located between $55^{\circ} \lesssim$ alt $\lesssim 75^{\circ}$ and $90^{\circ} \lesssim \mathrm{az} \lesssim 270^{\circ}$. It is interesting to note that the region with the lowest seeing $\left(0^{\prime \prime} 5\right)$ is not located at the highest elevation but rather in a region at $70^{\circ} \gtrsim$ alt $\gtrsim 65^{\circ}$ towards south. The region above this low seeing area shows an up to $0^{\prime \prime} \cdot 2$ stronger seeing, but is also covered by fewer observations. Note, that in Fig. 1 the contour indicating 500 data points per field shows a dip to $70^{\circ}$ in the south. This is because the ALT/AZ mount of the TMT site telescope. This might indicate that this patch of stronger seeing could be the result of an observational bias. In the following paragraph we show indications that this is probably not the case.

The two panels in Fig. 2 are similar to Fig. 1, but the data were selected according to the wind direction during the observations. While the top panel of Fig. 2 shows the skymap of the DIMM seeing during times when the winds are coming from the prevailing wind directions, ${ }^{8}$ i.e., $45^{\circ}-135^{\circ}$, the lower panel shows the sky under westerly winds, i.e., South-West-North $\left(180^{\circ}-360^{\circ}\right)$. It is obvious that the amount of observations under westerly winds is much lower. But the area between $75^{\circ}<$ alt $<80^{\circ}$ is strongest in both panels of Fig. 2. This indicates that indeed the high elevation strip shows a somewhat stronger DIMM seeing than the rest of the sky.

\section{A DIFFERENT VIEW ON MASS PROFILES}

The MASS reconstructs a turbulence profile $C_{n}^{2}(h) d h$ in six altitude bins at heights $h=0.5,1,2,4,8,16 \mathrm{~km}$, by measuring the spatial distribution of the scintillation in four concentric subapertures. The response function in each of these bins is approximately triangular and peaks at one at the given altitude and drops to zero at its neighboring altitudes. This leads to some slight overlap of adjacent layers. Creating sky maps from the MASS profiles similar to what was shown in Sec. 3 could in principle be done. However, here we look at these MASS profiles in a different way; in Fig. 3 our interpretation of the MASS profiles is shown. The air the MASS traces is - naturally - located along the line of sight. The $\sec z$ correction of the turbulence strength in each altitude bin converts this turbulence strength to zenith (eq. $9 \mathrm{in}^{1}$ ). Here we interpret this turbulence strength as the one which an observer - who is located at the same level of and at a distance of $h / \tan$ (alt) from the site monitor in the line of sight direction - would see towards zenith at height $h$. This interpretation opens the window on a new view on the spatial distribution of optical turbulence. Using a large sample of data collected over a long period of time, thus sampling a wide variety of atmospheric conditions, might allow to get a truly three dimensional view on the average spatial distribution of optical turbulence. Our approach is to compute for each MASS observation and each layer the projected distance from the site monitor and obtain the UTM (Universal Transverse Mercator) coordinates. As UTM coordinates for the $13 \mathrm{~N}$ location we used $240050 \mathrm{E}$ and $2194875 \mathrm{~N}$ in the $5 \mathrm{Q}$ zone. A large area around the site was then covered by a grid whose cell sizes could be adjusted for each layer. Each observation of a layer was then associated with the grid cell through which the line of sight passed at the time of observation. Then the median turbulence strength was computed from the observations in each grid cell. The resulting UTM maps of the median turbulence strengths (given as seeing $\epsilon$ ) at the altitudes of the MASS layers are shown in Figs. 4 and 6 to 8. These maps are overlaid on satellite images from GoogleEarth of the $13 \mathrm{~N}$ site. For the distance projection we used the nominal height of the MASS layers. This is reasonable for the 'pure' MASS layers, i.e., above $500 \mathrm{~m}$. But for the ground layer $\epsilon_{\mathrm{GL}}$ which is computed from the difference of MASS and DIMM seeing, $\epsilon_{\mathrm{GL}}=\left(\epsilon_{\mathrm{DIMM}}^{5 / 3}-\epsilon_{\mathrm{MASS}}^{5 / 3}\right)^{3 / 5}$, the situation is somewhat more complicated. This is because the DIMM response is one from the site monitor onwards. The combination with the MASS seeing 


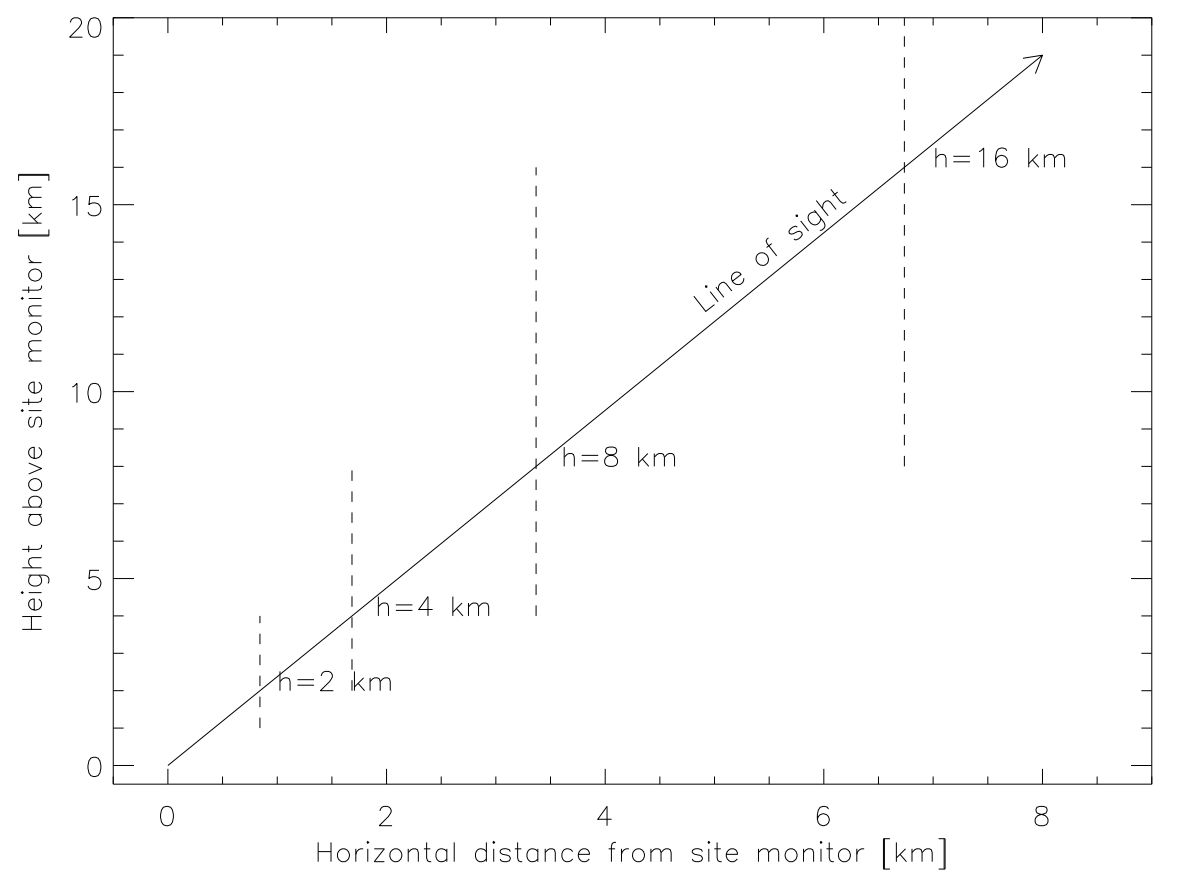

Figure 3. Sketch of how in the present work the MASS based turbulence profiles are interpreted. The traced air is along the line of sight (solid arrow). The MASS corrects this turbulence measurement to zenith, effectively showing the turbulence strength of the air along the dashed line. The dashed lines represent the vertical response of the MASS within each altitude bin (i.e., peaking at the nominal altitude $h$ and going down to zero at the neighboring altitudes). This is the turbulence strength above a location being at a projected distance from the site monitor. For displaying reasons the MASS altitude bins at $h=2,4,8,16 \mathrm{~km}$ are indicated only.

means that the response of the MASS-DIMM defined ground layer is one up to approximately $250 \mathrm{~m}$ and goes down to zero at $500 \mathrm{~m}$ (at the first MASS layer). Therefore, we used a nominal height of $h=250 \mathrm{~m}$ for the ground layer in the map in Fig. 4. One has to keep in mind, however, that a lot of weight of this layer is closest to the site monitor.

\subsection{The ground layer}

Despite this complication we will use the ground layer turbulence map in Fig. 4 to check numerical models of the turbulence distribution at the $13 \mathrm{~N}$ site. In Fig. 5 the result of a computational fluid dynamics model ${ }^{9}$ is shown. This Figure shows only the rms wind velocity which is indicative for the 'mechanical' turbulence component only. To properly compute seeing from those maps the thermal conditions would have to be included. Nevertheless, Fig. 5 indicates that stronger turbulence is to be expected when pointing towards the Mauna Kea summit ridge (or, from the summit itself towards South). As the vertical extend of Fig. 5 is less than $500 \mathrm{~m}$ and it represents particular conditions, it is only possible to do a qualitative comparison with the ground layer seeing map in Fig. 4. No major patches of strong velocity variance are seen in Fig. 5 in the air within $500 \mathrm{~m}$ around the $13 \mathrm{~N}$ site. This is - qualitatively - similar to what is seen in Fig. 4. Note, that the arc of strong $\epsilon_{\mathrm{GL}}$ ('white pixels') north of $13 \mathrm{~N}$ correspond to the strong seeing and poorly sampled arc discussed in Sec. 3.

\subsection{The higher layers}

Comparing the overall appearance of the maps shown in Figs. 4 and Figs. 6 to 8 it turns out that the optical turbulence in the $16 \mathrm{~km}$ layer appears most uniformly distributed of all maps. Compared to the other layers the observed 'strips' of the $16 \mathrm{~km}$ layer, despite being the widest spaced ones, are all showing within 0.'03 or $15 \%$ a similar turbulence strength. Only the western end pixels of some strips show a somewhat stronger seeing. 


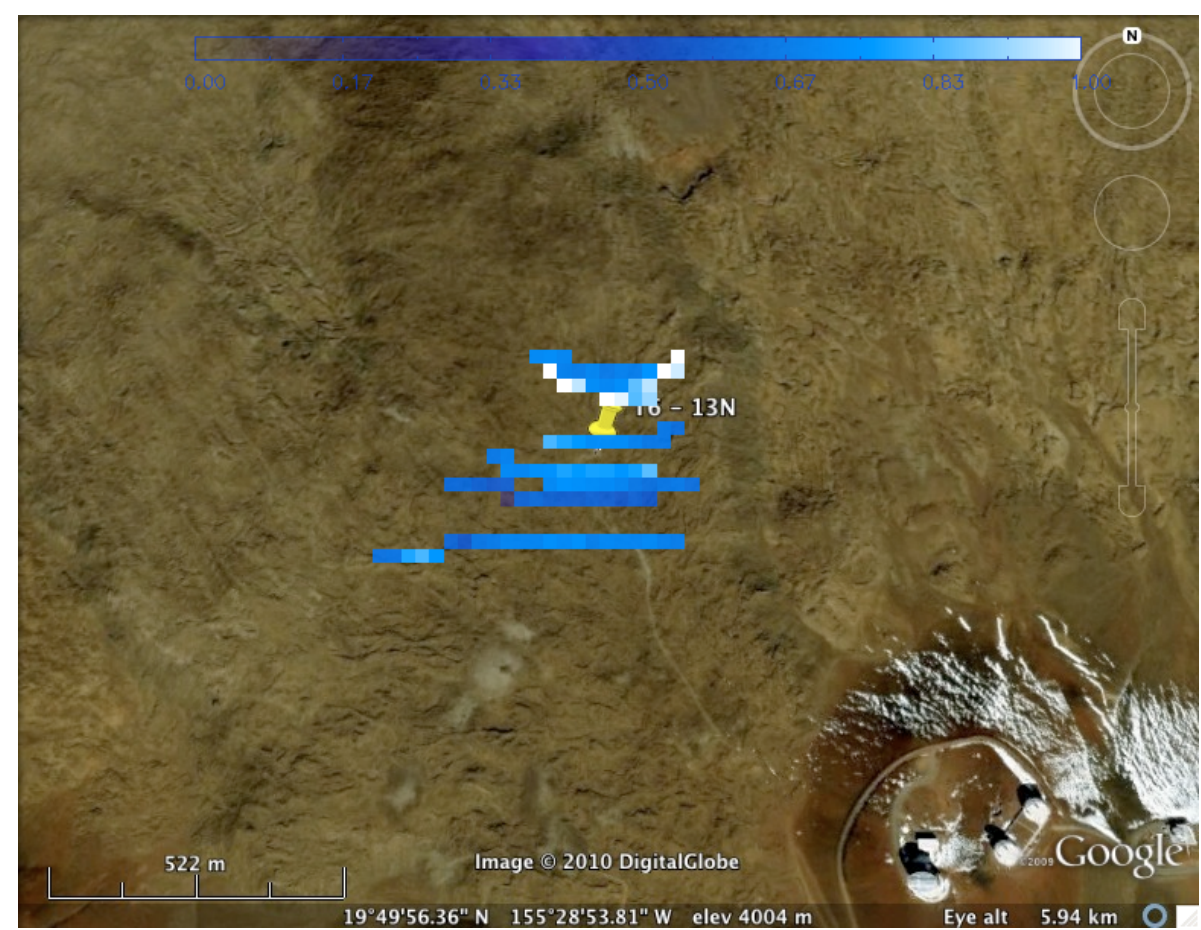

Figure 4. (color online) The horizontal turbulence map showing the median ground layer seeing $\epsilon_{\mathrm{GL}}, 25 \times 25 \mathrm{~m}^{2}$ pixels around the $13 \mathrm{~N}$ site (marked T6-13N). The color bar at the top indicates the range of $0^{\prime \prime}<\epsilon_{\mathrm{GL}}<1^{\prime \prime}$. A height of $250 \mathrm{~m}$ is assumed for the ground layer. The map is based on all data, i.e., no selection for particular meteorological conditions was done. The turbulence map is overlaid on the GoogleEarth satellite image available in GoogleEarth. North is up, East to the right. In the lower-right (south-east) corner the domes of the Keck and Subaru telescopes can be seen (note, that the white color in this region is snow and does not represent seeing).

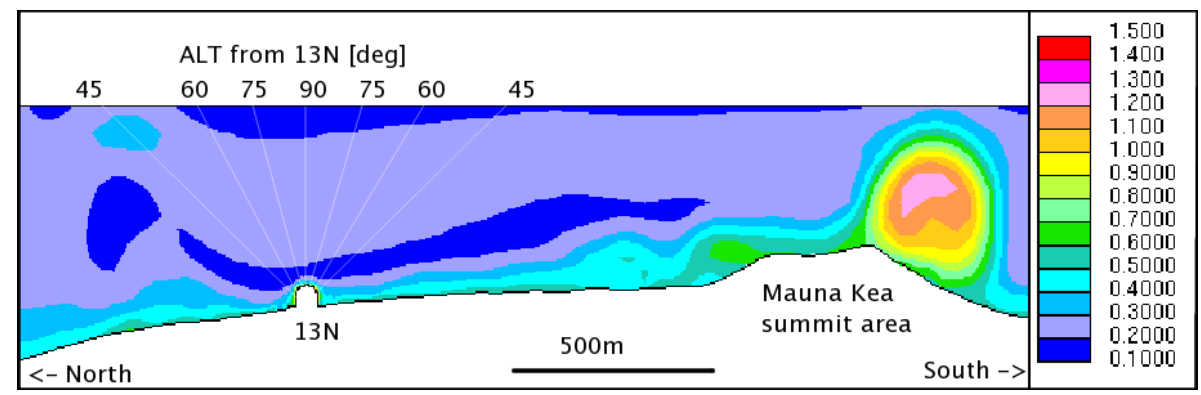

Figure 5. (color online) North-South cut of the summit area of Mauna Kea seen from the West, with the model of the dome of TMT on the $13 \mathrm{~N}$ site. This figure shows the computational area of the CFD simulations ${ }^{9,}{ }^{10}$ The color coding indicates model results of the $r m s$ wind speed in $\mathrm{m} / \mathrm{s}$. The wind coming from East, i.e., out of the image plane. The altitudes indicate several line of sights from $13 \mathrm{~N}$ through the atmosphere.

For the $8 \mathrm{~km}$ layer the strips show differences of up to 0.1 or $75 \%$. The high level of variability between the observed strips remains throughout the lower levels. Here we are cautious to not associate patches with stronger turbulence strengths to some underlying topographic features. Such a detailed study shall be presented in the future. Here we only point out that we do not believe that the high degree of uniformity of the $16 \mathrm{~km}$ layer is due to the very wide response function of that layer. If this would be the case the non-uniformity would successivly increase towards lower altitudes because the MASS layers become 'narrower'. But this is not the case as, e.g., the $4 \mathrm{~km}$ layer shows again a high degree of uniformity. 

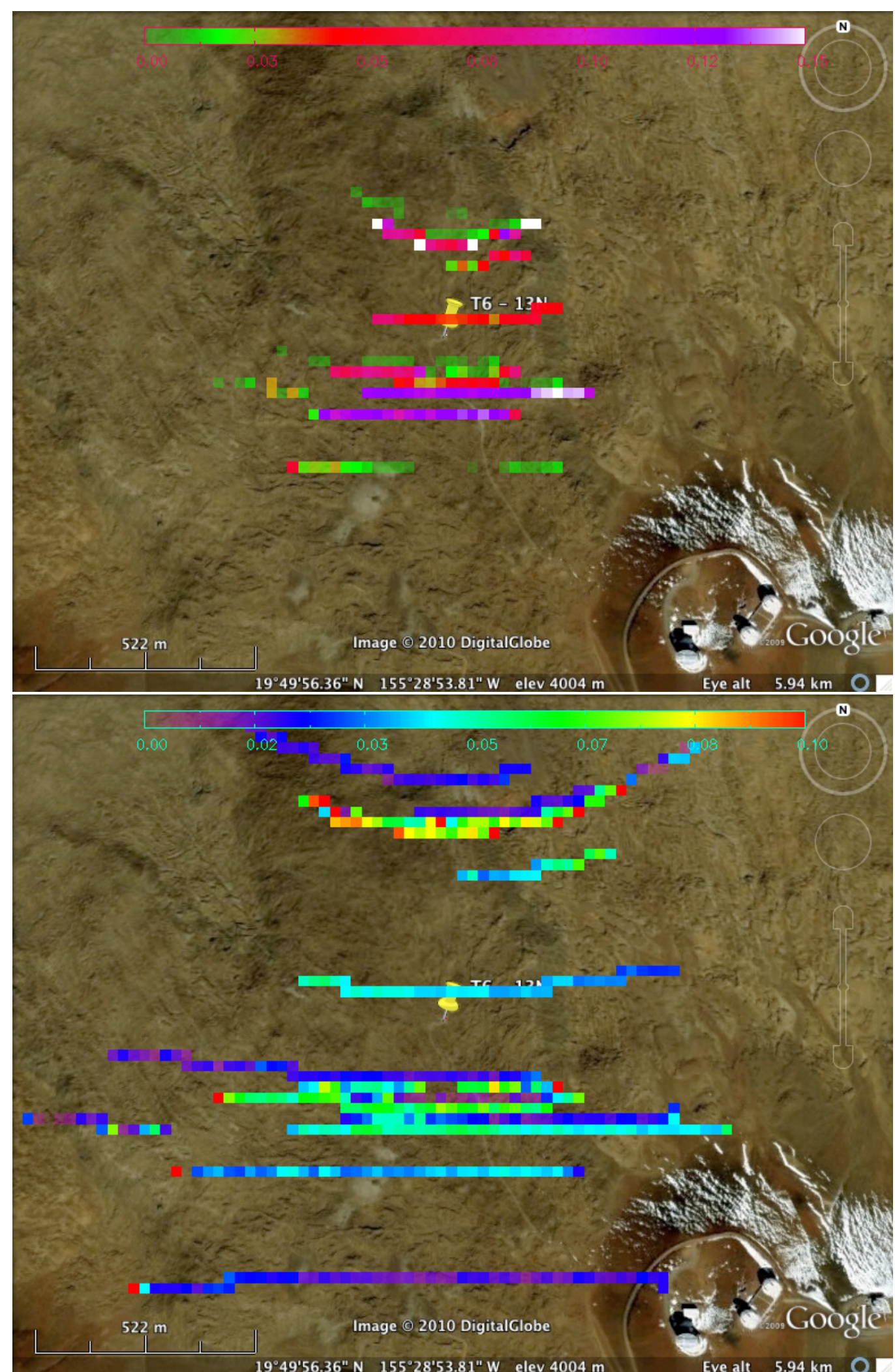

Figure 6. (color online) Horizontal turbulence maps of the median MASS layer seeing overlaid on the $13 \mathrm{~N}$ satellite image, similar to Fig. 4. Top panel: The $h=0.5 \mathrm{~km}$ MASS layer in $25 \times 25 \mathrm{~m}^{2}$ pixels. Colors indicate the range $0^{\prime \prime}<\epsilon_{\mathrm{GL}}<0^{\prime \prime} 15$. Lower panel: The $h=1 \mathrm{~km}$ MASS layer in $25 \times 25 \mathrm{~m}^{2}$ pixels. Colors indicate the range $0^{\prime \prime}<\epsilon_{\mathrm{GL}}<0$ "' 10 . 


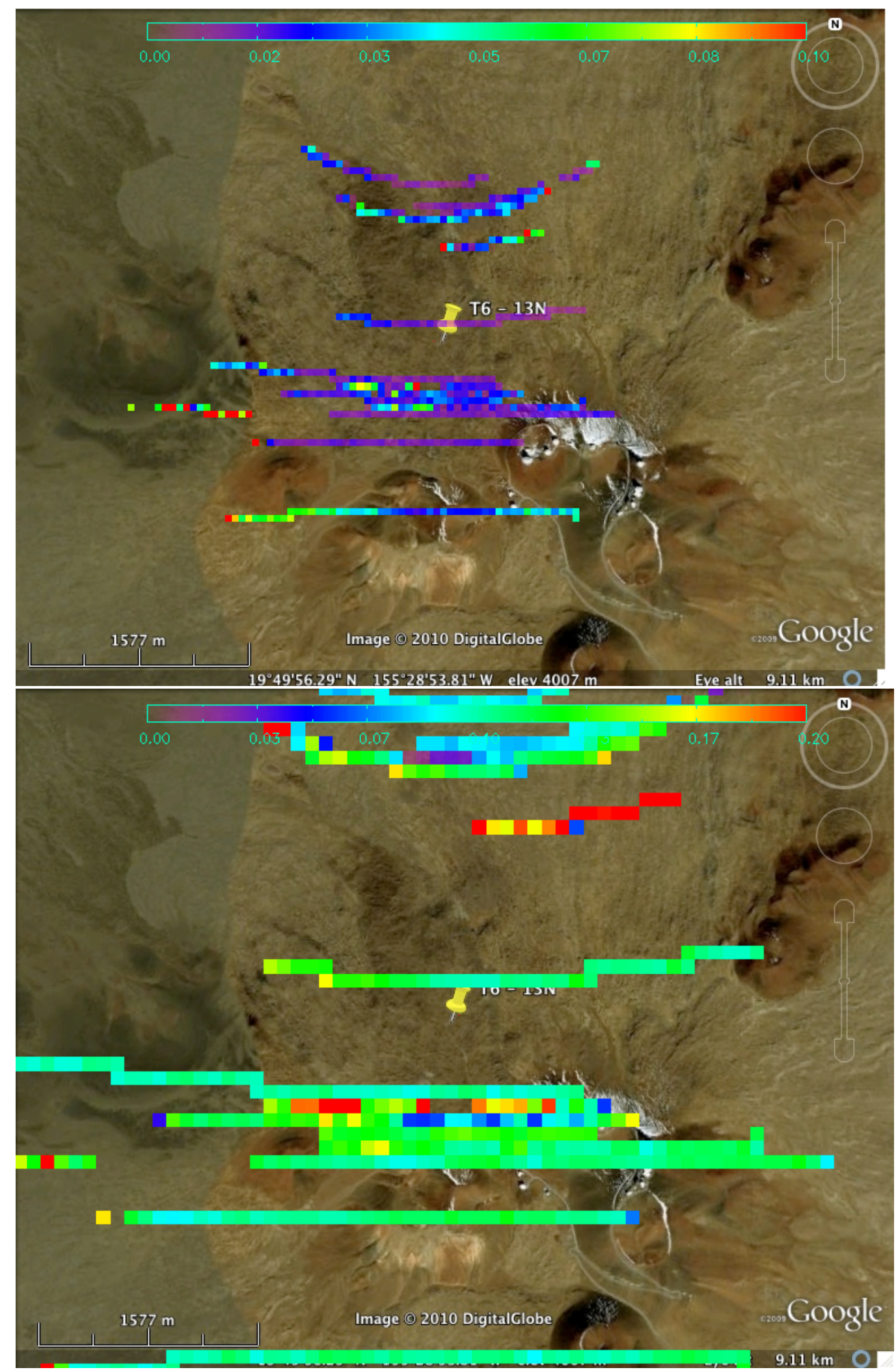

Figure 7. (color online) Horizontal turbulence maps of the median MASS layer seeing overlaid on the $13 \mathrm{~N}$ satellite image, similar to Fig. 4. Top panel: The $h=2 \mathrm{~km}$ MASS layer in $50 \times 50 \mathrm{~m}^{2}$ pixels. Colors indicate the range $0^{\prime \prime}<\epsilon_{\mathrm{GL}}<0^{\prime \prime} 10$. Lower panel: The $h=4 \mathrm{~km}$ MASS layer in $100 \times 100 \mathrm{~m}^{2}$ pixels. Colors indicate the range $0^{\prime \prime}<\epsilon_{\mathrm{GL}}<0^{\prime \prime} 20$. 

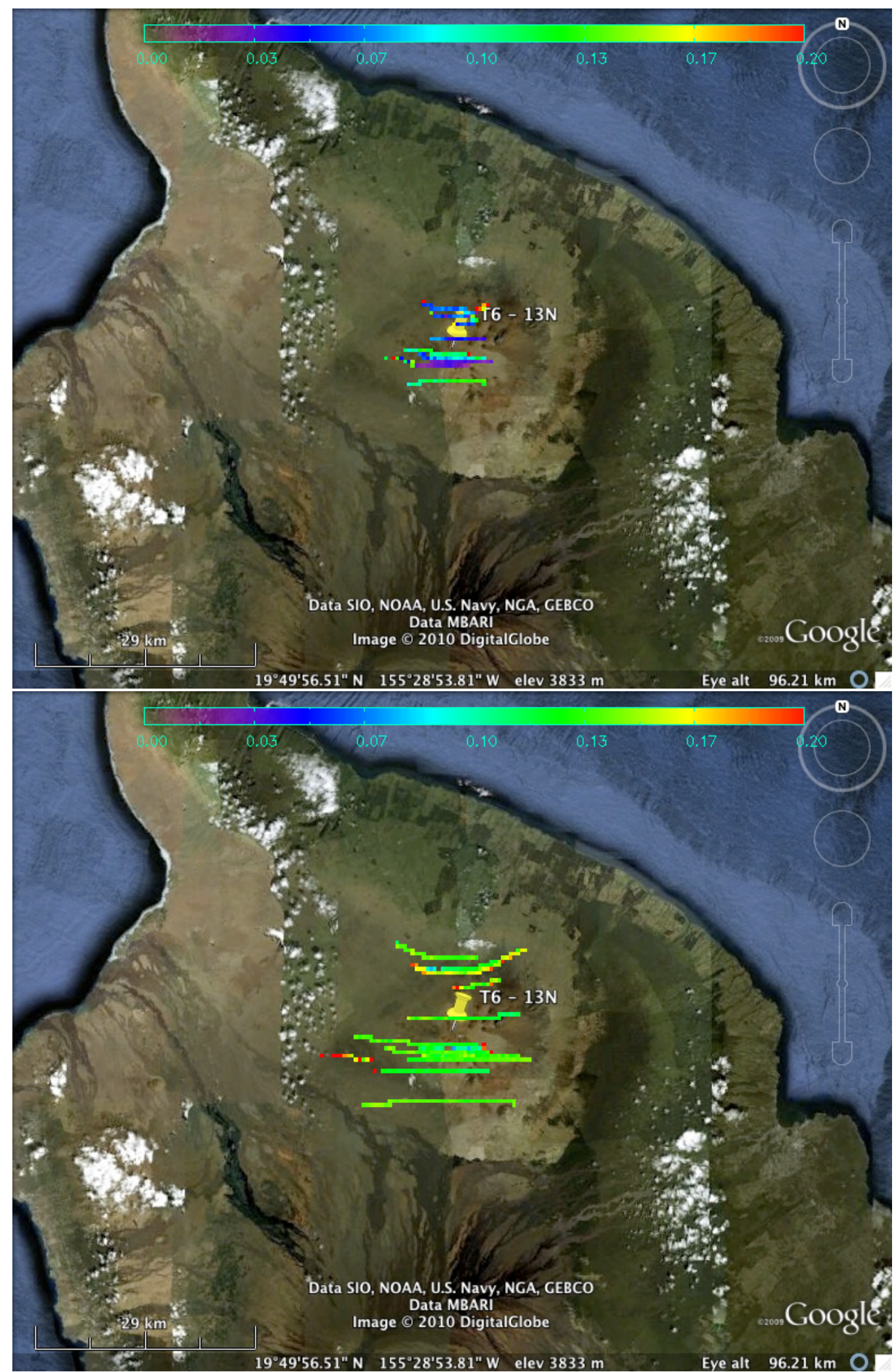

Figure 8. (color online) Horizontal turbulence maps of the median MASS layer seeing overlaid on the 13N satellite image, similar to Fig. 4. Top panel: The $h=8 \mathrm{~km}$ MASS layer in $100 \times 100 \mathrm{~m}^{2}$ pixels. Colors indicate the range $0^{\prime \prime}<\epsilon_{\mathrm{GL}}<0^{\prime \prime} 20$. Lower panel: The $h=16 \mathrm{~km}$ MASS layer in $100 \times 100 \mathrm{~m}^{2}$ pixels. Colors indicate the range $0^{\prime \prime}<\epsilon_{\mathrm{GL}}<0^{\prime \prime} .20$. 


\section{FUTURE TURBULENCE TOMOGRAPHY ?}

Despite Figs. 1 to 8 being already instructive, it is obvious that they only cover a very limited and patchy area above the site. This is not easy to overcome. Tuning the observing sequence and target star selection for the site monitors in a way that they cover a larger area more homogeneously across the sky, i.e., by observing more stars during the night, would increase the sky coverage. But this will also increase the observing overhead due to the more frequent star changes and it is only possible to a certain extend, as stars brighter than mag 3 are required. Still, a better sky coverage would ultimately result in filling in the terra incognita in our maps. Of course, this has to be done in a way that the unavoidable overheads for changing stars and re-pointing the telescope are minimized.

But is such a planning and monitoring effort useful ? For observatory operations a well sampled sky map of the seeing could help to improve scheduling. If areas which are prone to show stronger seeing do indeed exist above a site, a proper mapping of such regions could be used to avoid them when planning the observations of the most demanding and highly ranked observing programs. For example, the area of 'better seeing' at mid-altitudes towards South, identified in Sect. 3, could be of interest for programs observing objects in the vicinity of the black hole in the center of the galaxy. ${ }^{11}$

On the other hand this leads to a more fundamental question: what are the spatial scales on which optical turbulence does appear? It is up to now completely unknown whether areas of strong(er) optical turbulence appear preferably in patches, filaments or rather fronts. Also short lived sporadics boosting the optical turbulence during very few minutes along a particular line of sight can occur - or are these just the edges of the other geometries? We speculate that it will be a mix of all of those possibilities but that there will be differences from site to site on the preferred occurrence of these options (similar to the occurrence of clouds). Obviously, the maps shown in this article show the spatial turbulence distribution averaged over more than 2 years. Such average (median) maps can not trace the geometry of the areas of optical turbulence during short periods. To distinguish between these possible occurrences of optical turbulence some real time mapping of the sky would be required. The combination of MASS and DIMM appears to be the only suitable - or, economically sensible tool kit for such a setup. Being able to operate on small telescopes, a battery of $20 \mathrm{~cm}$ telescopes equipped with MASS-DIMMs could be used to monitor the turbulence distribution along numerous lines of sight. As the list of target stars for MASS is quite large in comparison to other turbulence monitoring techniques like SCIDAR, we can expect that always more than five stars could be observed at any site at all times. This setup would be able to trace the formation, movement and decay of turbulent areas in the sky. This would be an invaluable support to verify and improve numerical models which attempt to provide seeing forecasts.

To our knowledge there exist at present several setups of MASS-DIMM systems which resemble such a layout, but with fewer lines of sight. On Cerro Tololo two MASS-DIMMs are deployed side by side ${ }^{4}$ whose observations could also be linked to the site monitor(s) on Cerro Pachon. And on a wider length scale - few tens of $\mathrm{km}-$ also the data collected by the MASS-DIMM systems on Cerro Armazones, Cerro Paranal and Cerro Ventarrones could be used in combination. We have begun to look into the data collected in the Cerro Tololo and Paranal regions. If the results of these studies show interesting features, one should consider to aim for a dedicated instrument setup, as described in the previous paragraph.

\section{SUMMARY AND OUTLOOK}

Two different ways to display the spatial distribution of optical turbulence measured by MASS and DIMM have been presented. Both methods were implemented using the optical turbulence measurements collected by the TMT site testing project at the $13 \mathrm{~N}$ site on Mauna Kea. The first method displays the distribution of the DIMM seeing across the sky. Indications were found that the area between $55^{\circ} \lesssim$ alt $\lesssim 75^{\circ}$ and $90^{\circ} \lesssim$ az $\lesssim 270^{\circ}$ shows a weaker median seeing (by $\approx 0.2$ ) than the region towards higher elevations.

The second method re-projects the individual MASS layers back onto the horizontal locations where the measurements were taken. This allows to map the horizontal distribution of the optical turbulence at the height of the corresponding MASS layer. It was found that the highest MASS layer shows the most uniform distribution. All other layers show some stronger patchiness of the optical turbulence. 
The shown results have to be considered 'work in progress' and can at present only be interpreted in a qualitative manner. However, they demonstrate that optical turbulence monitoring could indeed result in a deeper understanding of the occurrence of atmospheric turbulence. It is at present already feasible to monitor the turbulence simultaneously along several lines of sight and thus perform real time mapping (with low spatial resolution) of the turbulent areas in the sky. This could become a field of investigation on its own; to map and identify the different geometries in which turbulence occurs above various locations. A proper experimental setup could then also improve scheduling of observing programs on large telescopes. And apart from being of interest as a basic science field it might be the only approach to properly verify (and understand) numerical models and prove (or disprove) the feasibility of seeing forecasts.

\section{ACKNOWLEDGMENTS}

This work is based on data collected during the site testing project for the Thirty Meter Telescope (TMT). The TMT Project gratefully acknowledges the support of the TMT partner institutions. They are the Association of Canadian Universities for Research in Astronomy (ACURA), the California Institute of Technology and the University of California. This work was supported as well by the Gordon and Betty Moore Foundation, the Canada Foundation for Innovation, the Ontario Ministry of Research and Innovation, the National Research Council of Canada, the Natural Sciences and Engineering Research Council of Canada, the British Columbia Knowledge Development Fund, the Association of Universities for Research in Astronomy (AURA) and the U.S. National Science Foundation.

\section{REFERENCES}

[1] Tokovinin A., Kornilov V., Shatsky N., Voziakova O., "Restoration of turbulence profile from scintillation indices", 2003, MNRAS, 343, 891

[2] Tokovinin A., "From Differential Image Motion to Seeing", 2002, PASP, 114, 1156

[3] Kornilov V., Tokovinin A., Shatsky N., et al., "Combined MASS-DIMM instruments for atmospheric turbulence studies", 2007, MNRAS, 382, 1268

[4] Els S.G., Schöck M., Bustos E., et al., "Four years of optical turbulence monitoring at CTIO", 2009, PASP, 121,922

[5] Masciadri E., Avila R., Sanchez L.J., "First evidence of the finite horizontal extent of the optical turbulence layers", 2002, A\&A, 382, 378

[6] Sanders G. \& Nelson J., "The status of the Thirty Meter Telescope Project", 2010, SPIE, 7733-69

[7] Schöck M., Els S., Riddle R., et al., "Thirty Meter Telescope Site Testing I: Overview", 2009, PASP, 121, 384

[8] Els S., Schöck M., Riddle R., Skidmore W., Travouillon T., "TMT Site Selection Results Update, April 2008", 2008, TMT Internal Report, available at http://sitedata.tmt.org/references.html

[9] Els S.G. \& Vogiatzis K., "TMT studies on the influence of short term changes of local thermodynamic conditions on the seeing: CFD vs. observations", 2006, SPIE 6267, 23

[10] Vogiatzis K., "Thermal Modeling Environment for TMT", 2010, SPIE, 7738, 11

[11] Ghez A., Salim S., Weinberg N.N., et al., "Measuring Distance and Properties of the Milky Way's Central Supermassive Black Hole with Stellar Orbits", 2008, ApJ, 689, 1044 